\title{
MASSIVE MURAL AORTIC ARCH THROMBUS.
}

\author{
Ali Ayaon Albarrán ${ }^{1}$, Jose Ignacio Juarez Del Rio ${ }^{1}$, María Mercedes Campos Sanz ${ }^{1}$, and \\ Cristina Rodríguez Alcalá \\ ${ }^{1}$ La Paz University Hospital. Madrid, Spain. \\ ${ }^{2}$ La Paz University Hospital. Madrid. Spain.
}

September 25, 2021

\begin{abstract}
Mural aortic thrombus is a rare pathology that is more frequently seen in severe atherosclerotic aortic walls, in aneurysms and acute aortic syndrome(1). However this can be found in patients without aortic disease, and be responsible for systemic or cerebral emboli. A 54-year-old male was admitted to our institution for syncope and aphasia, he was found in the street with ethylic intoxication. After neurological examination mixed type aphasia was observed, cerebral and supra aortic arteries CT angiography were performed. Cerebral CT showed focal filling defect of left middle cerebral artery. Supra aortic arteries CT angiography was completed with toracoabdominal CT because massive ascending and arch thrombus was found. The thrombus measured $130 \times 33 \times 15 \mathrm{~mm}$ (Figures $1 \mathrm{~A}$ and $1 \mathrm{~B}$ and $1 \mathrm{C}$ ), and covered from mid ascending aorta to $40 \mathrm{~mm}$ distal to the ostium of left subclavian artery. The patient was referred to our unit for urgent surgical treatment. Surgery was performed throw median sterntomy, cardiopulmonary bypass with moderate hypothermic arrest and anterograde cerebral perfusion via right axillary artery. Longitudinal aortotomy was made and 140 × 30 x $15 \mathrm{~mm}$ thrombus (Figure 2), attached to posterior mid ascending aorta, was found and resected, the aortic wall did not show any abnormality. The patient had an eventful recovery and was discharged 9 days later with oral anticoagulation and aspirin.
\end{abstract}

Subject category: Images in Cardiac Surgery

\section{TITLE:}

MASSIVE MURAL AORTIC ARCH THROMBUS.

Ali Ayaon Albarrán PhMD a* ${ }^{\text {a }}$ Ignacio Juarez del Rio MDa , María Mercedes Campos Sanz b MD, Cristina Rodríguez Alcalá MD ${ }^{\mathrm{b}}$.

a Adult Cardiac Surgery Department. La Paz University Hospital. Madrid. Spain.

b Anesthesiology Department, Division of Cardiothoracic Anesthesia, La Paz University

Hospital, Madrid, Spain

Running head : Massive aortic arch thrombus.

${ }^{*}$ Corresponding author. Adult Cardiac Surgery Department. La Paz University Hospital. Madrid. Spain. Paseo de la Castellana 261. 28046 - Madrid. Spain. Tel:(+34)91 72772 53; fax: $(+34) 9172772$ 53;email:ali.ayaon@salud.madrid.org(A. Ayaon Albarrán).

CONFLICT OF INTEREST: None declared.

Written informed consent was obtained from the participant of the study.

\section{Keywords}


Massive aortic arch thrombus * Circulatory arrest * Cardio pulmonary bypass *

\section{MAIN TEXT}

Mural aortic thrombus is a rare pathology that is more frequently seen in severe atherosclerotic aortic walls, in aneurysms and acute aortic syndrome(1). However this can be found in patients without aortic disease, and be responsible for systemic or cerebral emboli.

A 54-year-old male was admitted to our institution for syncope and aphasia, he was found in the street with ethylic intoxication. After neurological examination mixed type aphasia was observed, cerebral and supra aortic arteries CT angiography were performed. Cerebral CT showed focal filling defect of left middle cerebral artery. Supra aortic arteries CT angiography was completed with toracoabdominal CT because massive ascending and arch thrombus was found. The thrombus measured $130 \mathrm{x} 33 \mathrm{x} 15 \mathrm{~mm}$ (Figures 1A and $1 \mathrm{~B}$ and $1 \mathrm{C}$ ), and covered from mid ascending aorta to $40 \mathrm{~mm}$ distal to the ostium of left subclavian artery. The patient was referred to our unit for urgent surgical treatment. Surgery was performed throw median sterntomy, cardiopulmonary bypass with moderate hypothermic arrest and anterograde cerebral perfusion via right axillary artery. Longitudinal aortotomy was made and $140 \times 30 \times 15 \mathrm{~mm}$ thrombus (Figure 2), attached to posterior mid ascending aorta, was found and resected, the aortic wall did not show any abnormality. The patient had an eventful recovery and was discharged 9 days later with oral anticoagulation and aspirin.

\section{FIGURE LEGEND:}

Figure 1: Red star shows massive arch thrombus measuring $130 \times 33 \times 15 \mathrm{~mm}$ in chest CT-scan, axial view $(A)$; sagittal view (B); Coronal view $(C)$. CT:computed tomography.

Figure 2 : Intraoperative view after circulatory arrest and longitudinal aortotomy. Black star shows ascending aorta and aortic arch thrombus.

\section{FIGURES:}

Figure 1

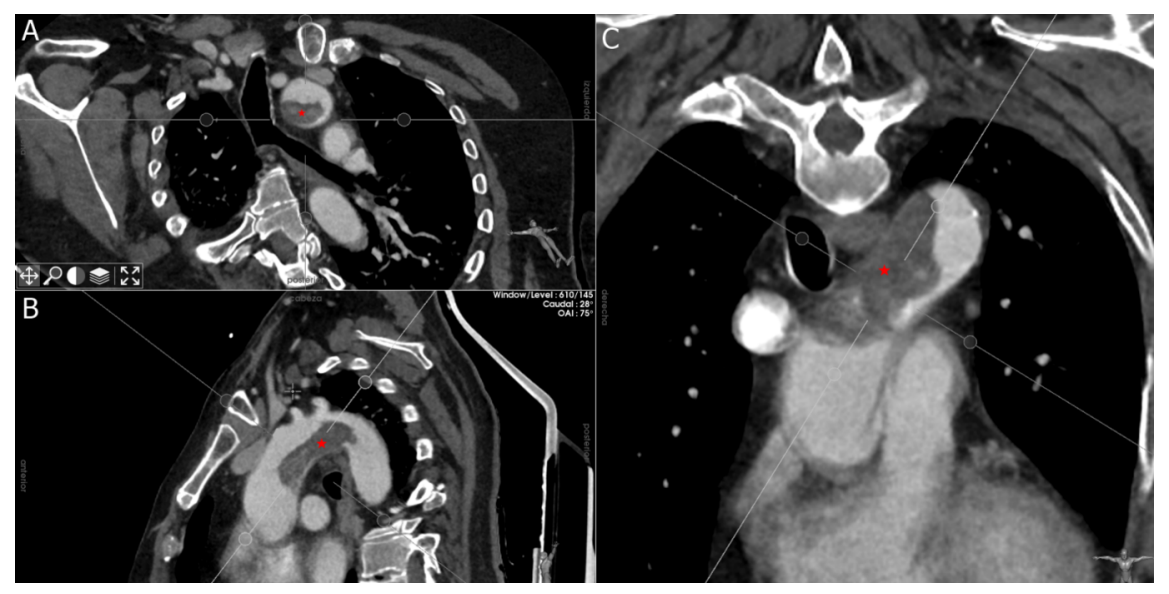

Figure 2 


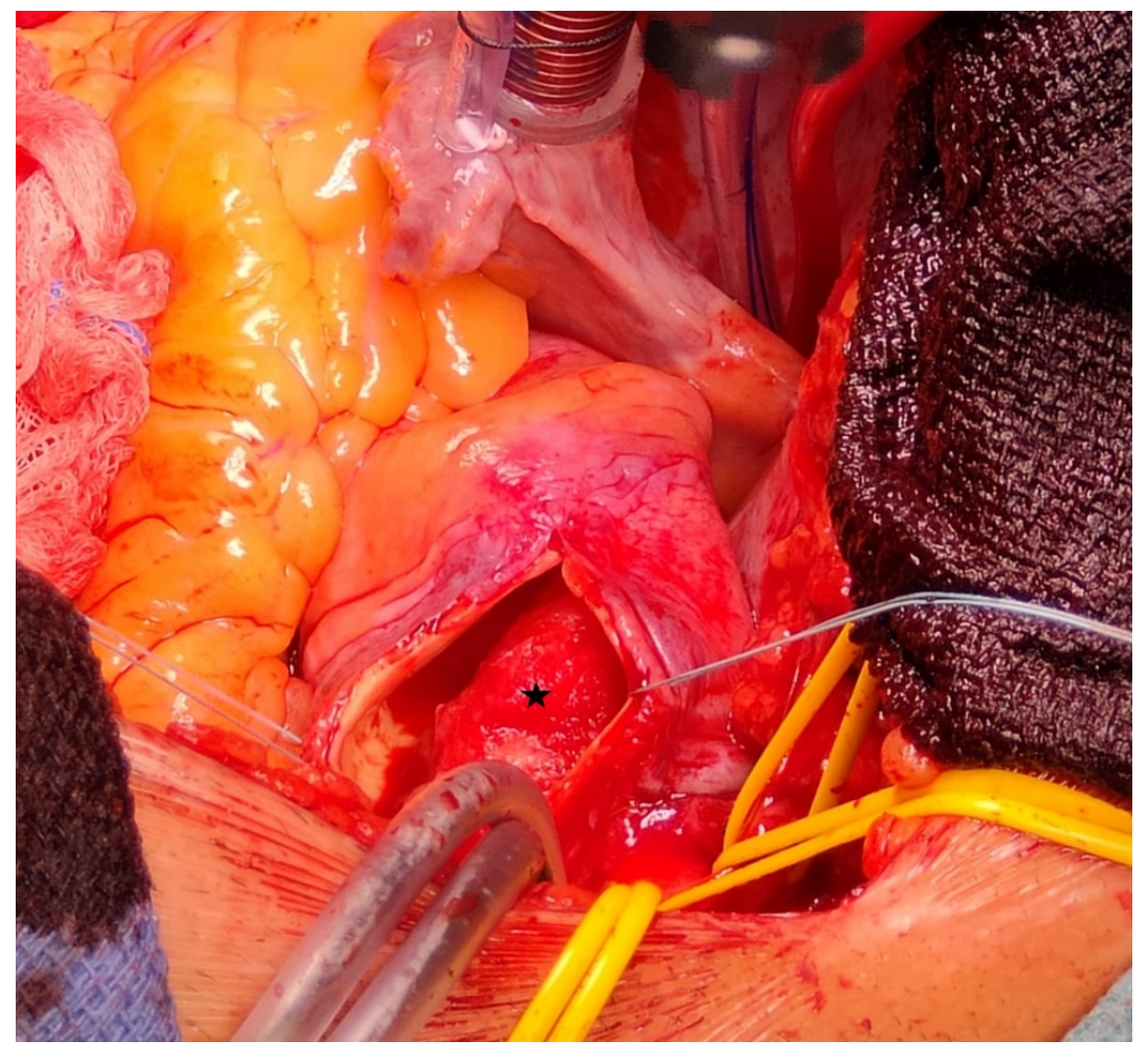

\section{REFERENCES:}

Fayad Z, Semaan E, Fahoum B, Briggs M, Tortolani A, D'Ayala M. Aortic mural thrombus in the normal or minimally atherosclerotic aorta. Ann Vasc Surg 2013;27:282-90.

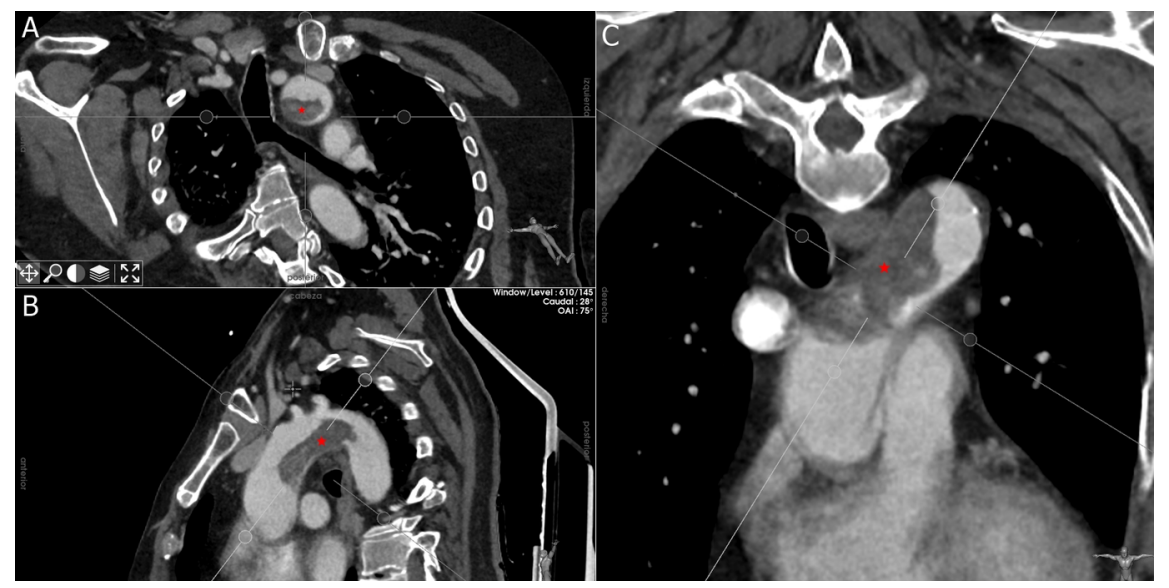




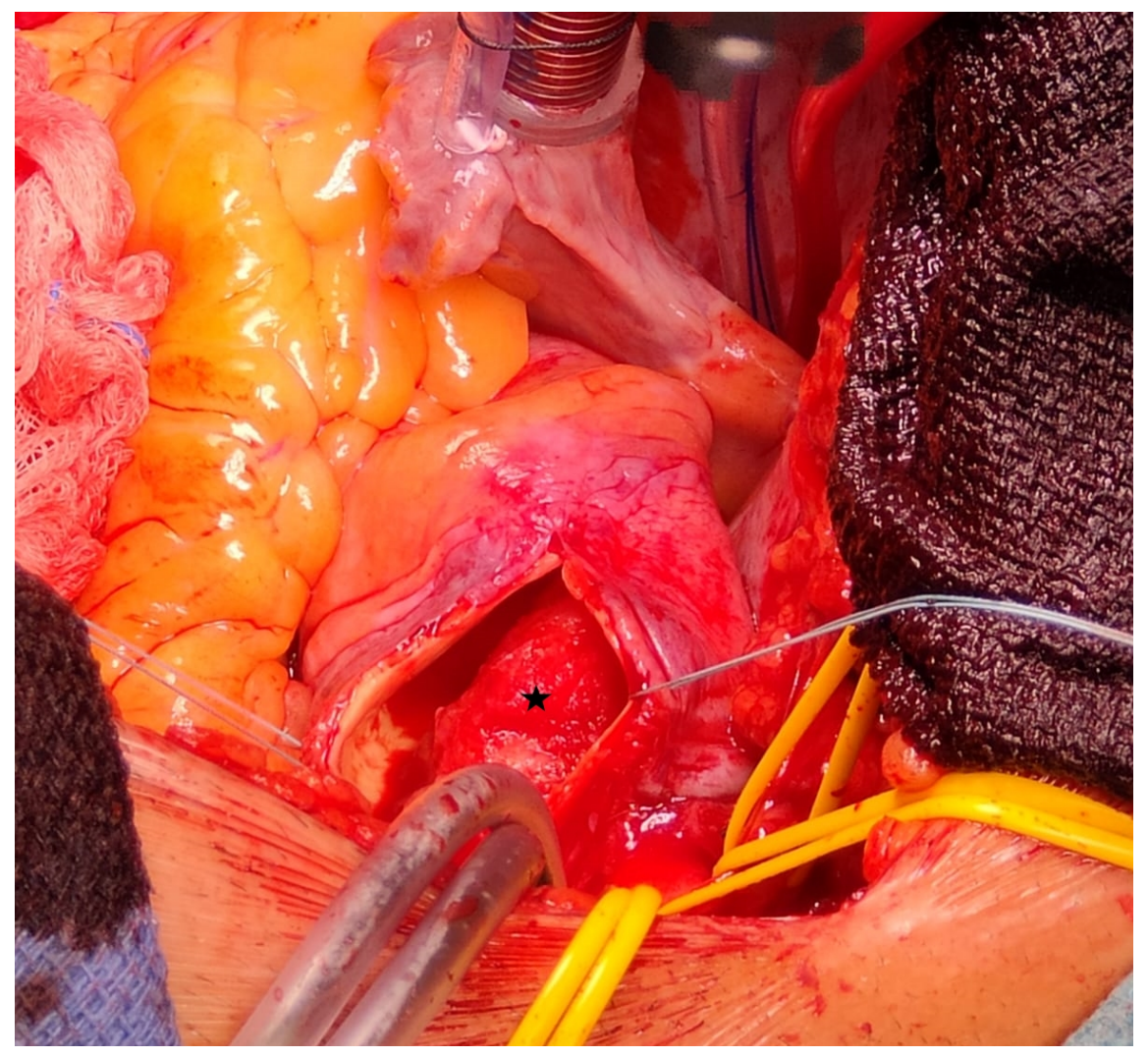

